\title{
Der Pflege-Bahr: \\ falsches Signal und untaugliches Geschäftsmodell
}

\section{Replik zu Biederbick und Weber}

KLAUS JACOBS, HEINZ ROTHGANG

Prof. Dr. Klaus Jacobs ist Geschäftsführer des Wissenschaftlichen Instituts der AOK (WIdO) in Berlin und Honorarprofessor an der Universität Duisburg-Essen

Prof. Dr. Heinz Rothgang ist Leiter der Abteilung Gesundheitsökonomie, Gesundheitspolitik und Versorgungsforschung des Zentrums für Sozialpolitik der Universität Bremen

\author{
Die Absicherung des wachsenden Pflegerisikos erfordert \\ stabile und nachhaltige Finanzierungsgrundlagen. \\ Die geförderte private Pflegezusatzversicherung - \\ der „Pflege-Bahr“ - taugt dazu nicht. Die folgende \\ Replik auf Annabritta Biederbeck und Roland Weber \\ zeigt, dass der Pflege-Bahr hinsichtlich Funktion und \\ Funktionsweise verfehlt und weitgehend ideologisch \\ begründet ist. Doch die Hauptverfechter dieser \\ Ideologie sind mittlerweile abgewählt, und der Irrweg \\ Pflege-Bahr könnte schnell wieder beendet werden.
}

Natürlich ist es grundsätzlich zu begrüßen, wenn über die Anfang 2013 eingeführte freiwillige private Pflegevorsorge mit steuerlicher Förderung - den Pflege-Bahr - fachlich diskutiert wird. Allerdings hätte das schon viel früher geschehen müssen. Stattdessen wurde der Pflege-Bahr im Sommer 2012 auf der Basis von Änderungsanträgen zu dem bereits im Gesetzgebungsprozess befindlichen Entwurf eines Gesetzes zur Neuausrichtung der Pflegeversicherung (PNG) mehr oder weniger „durchgepaukt“, und die nachfolgenden konkretisierenden Schritte, insbesondere die von der privaten Versicherungswirtschaft selbst vorgenommene Ausarbeitung der Mitte Dezember 2012 veröffentlichten „Musterbedingungen 2013 für die geförderte ergänzende Pflegeversicherung“, erfolgten weitgehend hinter geschlossenen Türen. Fundierte Warnungen von Wissenschaftlern und Verbraucherberatern an Funktion und Funktionsfähigkeit des Pflege-Bahr - etwa bei der öffentlichen
Anhörung im Gesundheitsausschuss des Deutschen Bundestags am 25.06.2012 (siehe dazu Ärzte Zeitung 2012) - blieben vollkommen unbeachtet, allein schon weil das Gesetz bereits vier Tage später am 29.06.2012 im Bundestag beschlossen wurde.

Grundsätzlich sollten bei der Diskussion über den Pflege-Bahr zwei Dimensionen auseinandergehalten werden: einerseits seine Funktion im Gesamtsystem zur Absicherung des Pflegerisikos, d.h. insbesondere im Zusammenspiel mit der gesetzlichen Pflegeversicherung (Soziale Pflegeversicherung und Private Pflegepflichtversicherung) und andererseits seine Funktionsweise, also die konkrete Ausgestaltung zur Erfüllung dieser Funktion.

\section{Funktion des Pflege-Bahr: falsches Signal}

Biederbick und Weber bezeichnen es in ihrem Beitrag als „unerheblich“, ob die private Pflegevorsorge gegenüber der ge- 
setzlichen Pflegeversicherung ergänzend oder substitutiv sei - entscheidend sei ihr privatrechtlicher Charakter. Diese Einschätzung mag zwar aus der Sicht eines privaten Versicherungsunternehmens verständlich sein, steht jedoch in krassem Gegensatz zur Auffassung der großen Mehrheit der Versicherten, die sich verbesserte Leistungen in der solidarisch finanzierten Pflegeversicherung wünscht (Zok 2011). Vor allem wird hier aber vom entscheidenden Punkt abgelenkt: der Rolle, die der Pflege-Bahr in der Gesamtarchitektur der finanziellen Absicherung des Pflegerisikos spielen kann und soll. Die Soziale Pflegeversicherung ist - aus guten Gründen - als Teilleistungssystem konzipiert. Das sagt zunächst aber noch nichts darüber aus, wie hoch der Anteil der Pflegekosten ist, der durch die Versicherungsleistungen gedeckt wird bzw. wie hoch die verbleibende Deckungslücke ausfällt. Sind die Versicherungsleistungen - auch in $\mathrm{Zu}$ kunft - im Vergleich zu den Pflegekosten „substanziell“, wie es die Bevölkerung mehrheitlich wünscht, relativiert sich automatisch die Notwendigkeit, spezielle Vorsorge für die verbleibende Deckungslücke zu treffen. Sind die Versicherungsleistungen dagegen im Vergleich zu den Kosten gering, und zwar - als Resultat einer unzureichenden Dynamisierung in Zukunft womöglich sogar in immer größerem Ausmaß, entsteht in der Tat eine Sicherungslücke, die aber durch den Pflege-Bahr nicht geschlossen werden kann, da der Nutzen der vereinbarten Leistungen der Zusatzversicherung vollkommen ungewiss ist. Wenn z.B. die Deckungslücke in 30, 40 oder 50 Jahren in einer Größenordnung jenseits von 2.000 Euro liegen sollte und die abzüglich der Leistung des Pflege-Bahr verbleibende Finanzierungslücke die Leistungsfähigkeit eines Rentners noch immer überfordert, nutzt eine private Vorsorge im Ergebnis allein dem Sozialhilfeträger. Genau aus diesem Grund findet etwa die Deutsche Bundesbank $(2012,9)$ „die Privatvorsorge für untere und mittlere Einkommensgruppen weniger attraktiv“. Niemand ist in der Lage, die Entwicklung der Pflegekosten, der Leistungen der gesetzlichen Pflegeversicherung und - nicht zuletzt - der eigenen Einkommenssituation über mehrere Dekaden hinreichend sicher einzuschätzen. Die Aussage, „ein bisschen“ private Vorsorge sei in jedem Fall besser als keine, ist deshalb für viele Versicherte schlicht unzutreffend. Einzig durch adäquate Leistungsdynamisierung der gesetzlichen Pflegeversicherung kann ein bestimmtes Sicherungsniveau auch langfristig garantiert werden.

Wie „erheblich“ die Frage „Ergänzung oder Substitution“ tatsächlich ist, zeigt gerade auch die von Biederbick und Weber selbst hergestellte Analogie zur Riester-Rente - allerdings nicht in Bezug auf die hehren Erwartungen bei ihrer Einführung, die in dem Zitat von Ulla Schmidt aus dem Jahr 2000 zum Ausdruck kommen, sondern angesichts der in der Zwischenzeit gesammelten Erfahrungen (z. B. Blank 2011, Kleinlein 2011). Die Rieser-Rente hat explizit substitutiven Charakter gegenüber der gesetzlichen Altersrente - mit dem Ergebnis, dass nur eine Minderheit der Förderberechtigten eine Riester-Rentenvertrag abgeschlossen hat, die Zahl der laufenden Verträge inzwischen ab- und nicht mehr zunimmt und große Teile der Bevölkerung auf ein Gesamtversorgungsniveau zusteuern, das merklich niedriger ausfällt, als wenn die gesetzliche Rentenversicherung im Hinblick auf ein substanzielles Sicherungsziel gezielt weiterentwickelt worden wäre. Altersarmut wird damit faktisch nicht „bekämpft“ (so die zitierte Behauptung von Ulla Schmidt), sondern ihr wird vielmehr geradezu erst der Boden bereitet (Schmähl 2011). Bei einem substitutiv wirkenden Pflege-Bahr wären die Folgen vom Grundsatz her vergleichbar, deshalb darf das Leistungsniveau der gesetzlichen Pflegeversicherung auf keinen Fall reduziert werden, sondern bedarf vielmehr des gezielten Ausbaus und einer regelgebundenen Dynamisierung (vgl. Rothgang/Jacobs 2013).

\section{Funktionsweise des Pflege- Bahr: untaugliches Modell}

Der Pflege-Bahr setzt aber nicht nur ein falsches Signal in Bezug auf die künftige Leistungsfähigkeit der gesetzlichen Pflegeversicherung, sondern ist überdies auch untauglich ausgestaltet und wird mit großer Wahrscheinlichkeit gar nicht dauerhaft funktionieren. Wenn Biederbick und Weber unter Verweis auf die Private Pflegepflichtversicherung (PPV) pauschal postulieren, dass „das Prinzip der privaten Pflegeversicherung “ funktioniere, unterschlagen sie dabei, dass die PPV und der Pflege-Bahr auf ganz unterschiedlichen Geschäftsmodellen basieren. Die PPV profitiert massiv von der Risikoselektion der privaten Krankenversicherung: Gegenüber der Sozialen Pflegeversicherung (SPV) sind die PPV-Versicherten durchschnittlich jünger und weisen zudem deutlich geringere altersspezifische Pflegehäufigkeiten auf. Deshalb waren in der PPV Ende 2011 lediglich 1,5 Prozent der Versicherten Leistungsempfänger, während dieser Anteil in der SPV mit 3,3 Prozent zum selben Zeitpunkt mehr als doppelt so hoch war (und sich inzwischen der 3,5-Prozent-Marke nähert). Vor diesem Hintergrund ist es kein Kunststück, vergleichsweise „erfolgreich“ zu sein, und hat mit dem privatwirtschaftlichen Charakter der PPV nicht das Geringste zu tun.

Die erheblichen Unterschiede in den altersspezifischen Pflegeprävalenzen der SPV- und PPV-Versicherten sind auch Gegenstand eines Papiers der Deutschen

\section{Der Pflege-Bahr ist untauglich ausgestaltet und wird mit großer Wahrscheinlichkeit nicht dauerhaft funktionieren.}

Aktuarvereinigung (DAV) von 2008. Dort heißt es, dass bei den (damals ausschließlich im Markt befindlichen) ungeförderten Pflegezusatzversicherungen „bei der Herleitung von Pflegeinzidenzen (...) Daten der privaten Pflegepflichtversicherung verwendet (werden), da davon auszugehen ist, dass die Zielgruppe für private Pflegeprodukte in der Lebensversicherung eher dem Kollektiv der privat als der sozial Pflegepflichtversicherten ähnelt. Dafür spricht einerseits, dass Versicherungsnehmer mit niedrigen verfügbaren Einkommen in der Regel nicht in der Lage oder bereit sein werden, die Beiträge für eine solche Zusatzdeckung zu bezahlen. Andererseits werden viele der Risiken, die zu der abweichenden Schadenerfahrung in der sozialen Pflegepflichtversicherung beitragen, aufgrund der vor Vertragsabschluss stattfindenden Gesundheitsprüfung in einem privaten Zusatzversicherungstarif nicht zu normalen Bedingungen versicherbar sein“ (DAV 2008, 13 f.). 
Beim Pflege-Bahr gibt es diesen Selektionseffekt aber nicht - vielmehr droht infolge der Vorschrift zur Aufnahmeverpflichtung ohne individuelle Risikozuschläge sowie des Parallelangebots ungeförderter Pflegezusatzversicherungen ohne Aufnahmeverpflichtung sogar eine „Antiselektion“ - zumindest sind die drei „Lehrbuch-Bedingungen“ für adverse Selektion sämtlich in Reinform erfüllt: erstens unterschiedliche Risiken in einer Population, zweitens die Kenntnis des eigenen Risikotyps seitens der Versicherten - insbesondere bei denen, deren Anträge auf ungeförderte Pflegezusatzversicherungen bereits abgelehnt oder wegen Aussichtslosigkeit gar nicht erst gestellt wurden - und drittens die Unmöglichkeit (hier: das Verbot) der Versicherer, diese Risiken adäquat $\mathrm{zu}$ tarifieren (siehe Jacobs/Rothgang 2012, 47).

Biederbeck und Weber halten das Problem der adversen Selektion für überbetont und verweisen darauf, dass die Versicherer sehr wohl die Möglichkeit hätten, der entsprechenden Gefahr entgegenzuwirken, etwa indem sie den Abschluss einer nicht geförderten Pflegevorsorge an den Abschluss eines PflegeBahr knüpfen. Aber welcher Versicherer - außer vielleicht der Debeka - nutzt diese Option tatsächlich konsequent, und wird sie am Ende genügen, um nennenswerte Beitragssteigerungen zu verhindern? Ein Großteil der gesunden Versicherten, die überhaupt an einer privaten Pflege-Zusatzversicherung interessiert sind, verfügt darüber ja heute schon ohne Verknüpfung mit dem Pflege-Bahr. Außerdem ist die adverse Selektion für die Versicherer selbst insofern irrelevant, als sie ihre Tarife ausdrücklich nachkalkulieren dürfen. Ins Leere läuft darüber hinaus auch die von Biederbeck und Weber geäußerte Erwartung, dass das Interesse an der staatlichen Förderung dem Pflege-Bahr ,per Mitnahmeeffekt“ $\mathrm{zu}$ einer ausgewogenen Mischung von Gesunden und Kranken verhelfe - zumindest solange der Prämienunterschied zwischen geförderten und nicht geförderten Versicherungen den Umfang der staatlichen Förderung von monatlich 5 Euro übersteigt.

Verbraucherberater - der Bund der Versicherten (BdV 2013) ebenso wie der Verbraucherzentrale Bundesverband (vzbv 2012) und die Stiftung Warentest (2013) - raten Personen ohne Behinde- rungen und Vorerkrankungen jedenfalls ausdrücklich vom Pflege-Bahr ab. Damit verstärken sie naturgemäß die Gefahr der adversen Selektion, deren Folgen in jedem Fall allein die Versicherten zu tragen haben. Sollten sich die Beiträge angesichts der Anzahl der eintretenden Leistungsfälle als zu niedrig erweisen - mit welchen Annahmen die jetzigen Tarife kalkuliert sind, ist ohnehin nicht bekannt -, werden sie entsprechend erhöht. Allerdings wird dies - allein schon wegen der fünfjährigen Karenzzeit - erst in einigen Jahren geschehen, und dann sind die Versicherten praktisch an ihren Versicherer „gefesselt“. Das ihnen ausdrücklich eingeräumte Kündigungsrecht bei Beitragserhöhungen ist letztlich eine Farce, weil die zuvor gebildeten Alterungsrückstellungen (einschließlich der staatlichen Förderung) bei einer Kündigung verlorengehen. Und anders als bei der Riester-Rente, bei der mittlerweile fast jeder fünfte Vertrag ,ruhend gestellt“ ist, verfallen beim Pflege-Bahr sämtliche Leistungsansprüche, wenn die Beiträge - mit oder ohne formelle Kündigung - nicht mehr gezahlt werden (mit Ausnahme einer dreijährigen Ruhenszeit bei Versicherten mit nachgewiesener Hilfebedürftigkeit).

Wer sich etwa für ein besonders günstig anmutendes Angebot zum PflegeBahr entscheidet, das aufgrund einer mit oder ohne Vorsatz - unrealistischen Kalkulation schon vergleichsweise bald spürbare Beitragssteigerungen erfordert, wird zum Opfer des Geschäftsmodells

\section{Wer sich für den Pflege- Bahr entscheidet, wird zum Opfer des Geschäftsmodells der privaten Kranken- und Pflegeversicherung, bei dem der Wettbewerb auf Neuabschlüsse beschränkt ist.}

Altersrückstellungen beim Pflege-Bahr noch zu einem Zeitpunkt eingeführt wurde, zu dem selbst der Gesundheitsminister öffentlich darüber sprach, den Wettbewerb um Bestandsversicherte in der privaten Krankenversicherung durch die Möglichkeit zur vollständigen Mitgabe der Alterungsrückstellungen in Gang zu setzen (FAZ 2013). Der Pflege-Bahr hätte leicht von Anfang an so ausgestaltet werden können.

\section{Résumé}

In einem Punkt scheint es immerhin Konsens zu geben, nämlich in der Skepsis gegenüber der Verlässlichkeit der $\mathrm{Zu}$ sagen von Staat und Politik. Aber schon bei der Schlussfolgerung aus dieser Diagnose tritt wieder ein grundlegender Dissens zutage, denn Skepsis gegenüber dem Staat als Gewährleistungsträger in der umlagefinanzierten Sozialen Pflegeversicherung bedeutet ja keineswegs automatisch Zutrauen in die von Biederbeck und Weber aufgezeigte Alternative: das im Hinblick auf sich ändernde Anforderungen insbesondere über längere Zeiträume weithin anpassungsunfähige Kapitaldeckungsverfahren, das zudem eine andere, mindestens ebenso problematische Abhängigkeit aufweist, nämlich vom Finanzmarkt - und da mag jeder selbst entscheiden, in welchem Fall größere Skepsis angebracht erscheint. Weil zumindest staatliches Versagen keineswegs unvermeidlich ist, sollten alle Anstrengungen darauf ausgerichtet werden, den „Willkürfaktor" im öffentlichen System durch klare Regelvorgaben - insbesondere zur regelmäßigen substanziellen Anpassung der Leistungssätze der gesetzlichen Pflegeversicherung an die Entwicklung der Pflegekosten - möglichst weit auszuschalten. Eine derartige Forderung wäre die angemessene Reaktion auf die Zweifel an der Verlässlichkeit der $\mathrm{Zu}$ sagen von Staat und Politik.

der privaten Kranken- und Pflegeversicherung, bei dem der Wettbewerb mangels umfassender Portabilität der Alterungsrückstellungen weitgehend auf Neuabschlüsse beschränkt ist (siehe Jacobs 2013). Es ist schwer verständlich, dass ein derart wettbewerbsfeindliches Konzept ohne jegliche Portabilität der
Aber selbst wer unverdrossen auf die Ergänzung der umlagefinanzierten Sozialversicherung durch Kapitaldeckung setzt, kann im Pflege-Bahr kein taugliches Instrument erkennen. So hat sich etwa der Vorstand der Deutschen Gesellschaft für Gesundheitsökonomie zur Sicherung der Funktionsfähigkeit einer 
ergänzenden privaten Pflegevorsorge für deren obligatorische Ausgestaltung ausgesprochen (DGGÖ 2012). Dass die Funktionsfähigkeit einer freiwilligen Pflegevorsorge mit Aufnahmeverpflichtung, aber ohne risikobezogene Tarifierung aufgrund der Unvermeidbarkeit von adverser Selektion ernsthaft in Frage steht, hätten die deutschen Verantwortlichen auch von ihren USamerikanischen Kollegen lernen können. Dort hatte man nämlich gerade erst nach mehrmonatigen aktuariellen Berechnungen im Gesundheitsministerium die zuvor bereits vom Parlament beschlossene Einführung einer freiwilligen Pflegeversicherung schweren Herzens „storniert", weil sich die gestellten Anforderungen - substanzielle Versicherungsleistungen bei langfristig bezahlbaren Beiträgen - unter diesen Bedingungen als nicht realisierbar erwiesen (vgl. Jacobs/Rothgang 2012, von Schwanenflügel 2012).

Insgesamt markiert der Pflege-Bahr ein trauriges Kapitel deutscher Sozialgeschichte. Immerhin scheint er zumindest bislang bei weitem nicht so erfolgreich zu sein, wie er seitens der verantwortlichen
Politiker und der privaten Versicherungswirtschaft - auch von Biederbeck und Weber - ausgegeben wird. Laut Gesetzesbegründung wurde Mitte 2012 „angenommen, dass im Jahr 2013 etwa 1,5 Millionen förderfähige Versicherungsverträge abgeschlossen werden" (Ausschuss für Gesundheit 2012, 43), doch vermeldete der PKV-Verband kurz vor Jahresmitte 2013, dass zu diesem Zeitpunkt die Marke von 150.000 Verträgen angesteuert werde (PKV 2013), also gerade einmal 10 Prozent der projektierten Zahl nach der Hälfte der Zeit. Anfang September 2013 ging PKV-Verbandschef Uwe Laue davon aus, dass bis Ende 2014 (!) eine Million staatlich geförderte Verträge verkauft werden (Ärzte-Zeitung online 2013) - eine wirkliche Erfolgsstory sieht anders aus.

Deshalb ist es grundsätzlich positiv zu bewerten, dass die Unionsparteien in ihrem ansonsten mit konkreten Aussagen nicht gerade gespickten Programm zur Bundestagswahl ausdrücklich das Ziel der Sicherstellung eines hohen Niveaus der Pflegeleistungen formuliert haben und „einen steigenden Zuzahlungsbedarf zulasten der Pflegebedürftigen, ihrer An- gehörigen und der Sozialhilfeträger verhindern (wollen)“ (CDU/CSU 2013, 78). Offenbar hat auch die Union erkannt, dass sich eine gerechte und nachhaltige Pflegepolitik an den Interessen und Bedürfnissen der Mehrheit der Bevölkerung und nicht an den Wünschen der privaten Versicherungswirtschaft orientieren sollte. Denn nur so kann das wachsende Pflegerisiko in Deutschland abgesichert werden: durch substanzielle Leistungen in der von der gesamten Bevölkerung solidarisch finanzierten Sozialen Pflegeversicherung.

\section{Literatur}

Ärzte Zeitung (2012): Fachleute lassen kein gutes Haar am „Pflege-Bahr“, Ärzte Zeitung vom 26.06.2013, 5

Ärzte Zeitung online (2013): PKV-Unternehmen: Zusatzpolicen bleiben Zubrot, Download unter: www.arztezeitung.de (Zugriff: 10.09.2013).

Ausschuss für Gesundheit des Deutschen Bundestags (2012): Änderungsantrag 18 der Fraktionen der CDU/CSU und FDP zum Entwurf eines Gesetzes zur Neuausrichtung de Pflegeversicherung. Ausschuss-Drucksache 17(14)287 vom 12.06.2012.

BdV [=Bund der Versicherten] (2013):

Merkblatt - „Pflege-Bahr“: Staatlich geförderte, private Pflegezusatzversicherung,

Stand: 12.04.2013; Download unter: www.bundderversicherten.de/Kanken-Pflege/ Pflege-Bahr (Zugriff: 05.08.2013)

Blank F (2011): Die Riester-Rente: ihre Verbreitung, Förderung und Nutzung. Soziale Sicherheit 60 (12), 414-420.

CDU/CSU (2013): Gemeinsam für Deutschland Regierungsprogramm 2013-2017; Download unter: www.cdu.de/regierungsprogramm (Zugriff: 05.08.2013).

DAV [=Deutsche Aktuarvereinigung] (2008): Herleitung der Rechnungsgrundlagen DAV $2008 \mathrm{P}$ für die Pflegerenten(zusatz)versicherung der DAV-Unterarbeitsgruppe „Rechnungsgrundlagen der Pflegeversicherung“;
Download: https://aktuar.de/custom/ download/dav/veroeffentlichungen/ 2008-12-04-Herleitung-der-DAV-2008P.pdf (Zugriff: 05.08.2013)

DGGÖ [=Deutsche Gesellschaft für Gesundheitsökonomie] (2012): Für eine verpflichtende private Pflegevorsorge mit Zuschüssen für Bedürftige. Stellungnahme der DGGÖ vom 21.06.2013

Download: http://file.dggoe.de/presse/ 2012-06-19-Pflegereform-StellungnahmeDGG\%C3\%96.pdf (Zugriff: 05.08.2013).

FAZ [=Frankfurter Allgemeine Zeitung] (2013): „Ich will mehr Wettbewerb in der privaten Krankenversicherung“. Im Gespräch: Daniel Bahr (FDP), Bundesminister für Gesundheit. Frankfurter Allgemeine Zeitung vom 08.07.2013, 19.

Jacobs K (2013): Wettbewerb im dualen Krankenversicherungssystem in Deutschland - Fiktion und Realität. In: Jacobs K, Schulze S (Hrsg.): Die Krankenversicherung der Zukunft. Anforderungen an ein leistungsfähiges System. Berlin: KomPart, 47-73.

Jacobs K, Rothgang H (2012): Der Pflege Bahr: zum Schaden der Sozialen Pflegeversicherung und ihrer Versicherten. Gesundheits- und Sozialpolitik 66 (5), 41-51.

Kleinlein A (2011): Zehn Jahre „Riester-Rente“. Bestandsaufnahme und Effizienzanalyse. Expertise im Auftrag der Abteilung Wirt- schafts- und Sozialpolitik der Friedrich-EbertStiftung, Bonn; Download: http://library.fes. de/pdf-files/wiso/o8683.pdf (Zugriff: 05.08.13)

PKV [=Verband der privaten Krankenversicherung] (2013): Große Nachfrage nach geförderter Pflegevorsorge - gerade auch bei jungen Leuten. Pressemitteilung vom 19.06.2013, Berlin.

Rothgang H, Jacobs K (2013): Pflegereform 2014: Was ist zu tun? G+G Wissenschaft 13 (3), 7-14.

Schmähl W (2011): Die Riester-Reform von 2001 - Entscheidungen, Begründungen, Folgen. Soziale Sicherheit 60 (12), 405-414 Schwanenflügel M von (2012): Einsichten in die ministerielle Arbeit in Washington. Gesundheits- und Sozialpolitik 66 (1), 23-28 Stiftung Warentest (2013): Lieber ohne Förderung. Finanztest, Heft 5/2013, 70-77. vzbv [=Verbraucherzentrale Bundesverband] (2013): Die Zusatzversicherung für Pflege eine sinnvolle Ergänzung? Download unter www.vzbv.de/10943.htm (Zugriff: 05.08.2013).

Zok K (2011): Erwartungen an eine Pflegereform. Ergebnisse einer Repräsentativbefragung. WIdOmonitor 8 (2); Download unter: www.wido.de/wido_monitor_2_2011.html (Zugriff: 05.08.13). 